\title{
Hemi-dystonia secondary to localised basal ganglia tumour
}

\author{
J NARBONA, ${ }^{*}$ JA OBESO, ${ }^{*}$ T TUÑON, $\dagger$ JM MARTINEZ-LAGE, ${ }^{*}$ CD MARSDEN. $\ddagger$ \\ From the Departments of Neurology (Neuropediatric and Movement Disorders Unit) ${ }^{*}$ and Pathology, $\dagger$ \\ Clinica Universitaria, University of Navarra Medical School, Pamplona, Spain, and University Department of \\ Neurology, Institute of Psychiatry and King's College Medical School, London, England. $\ddagger$
}

SUMMARY An 8-year-old boy with an 18 month history of left limb hemi-dystonia due to a right lenticular nucleus astrocytoma originating in the putamen is reported. Subsequent neuropathological study demonstrated that the tumour was mainly localised to the right lenticular nucleus, with cystic necrosis in the infero-lateral putamen. Solid tumour also infiltrated the right hypothalamus, the anterior commisure and the optic chiasm, and there was perivascular spread into the globus pallidus, internal capsule and roof of the right lateral ventricle. This case, and the few other published reports of symptomatic dystonia due to focal brain lesions verified pathologically, indicate that damage to the lenticular nucleus, and to the putamen in particular, can cause limb dystonia in man.

Torsion dystonia is a clinical syndrome characterised by prolonged spasms of muscle contractions, which distort the body into typical dystonic postures. The pathological or biochemical basis of either the familial types or of most sporadic forms of idiopathic or primary torsion dystonia is unknown.' There are numerous reports of patients with symptomatic or secondary dystonia but such dystonia usually has been associated with widespread pathological lesions in the brain. ${ }^{2}$ Cases of pure focal dystonia due to a localised brain lesion with pathological confirmation are rare. We report the clinical features and pathological findings in a patient with hemi-dystonia secondary to an astrocytoma originating in the putamen.

\section{Case report}

An 8-year-old right-handed boy presented in August 1979 with a 4 month history of abnormal postures and involuntary movements of the left limbs. He was the product of a normal pregnancy and delivery, and his early development and general health had been excellent until this recent illness. His parents were healthy and unrelated, and there was no history of any similar illness in any other member of the family.

Address for reprint request: Dr JA Obeso, Neurologia, Clínica Universitaria, Apartado 192. Pamplona, Spain.

Received 28 November 1983. Accepted 5 January 1984
The illness commenced with the left hand adopting bizarre, unwanted postures on attempted action. Gradually all manual activity became distorted by such involuntary movements, and drawing with the left hand became increasingly difficult. Three months after the onset of symptoms in the left hand, the left foot also became affected, beginning to plantar-flex and invert on walking.

On examination in August 1979, the cranial nerves and right limbs were normal. Any slight body movement provoked the left arm to adopt a dystonic posture, with the wrist assuming a posture of ulnar deviation and slight flexion with the fingers extended. The outstretched left arm also adopted a similar posture, with additional excessive pronation. Dystonia prevented any useful action of the left arm. At rest the left foot was inverted and plantar flexed, with a tendency for the left big toe to adopt a spontaneous dystonic extension. This dystonic posture of the left foot was exaggerated when he tried to walk. Passive movement of wrist or ankle provoked a springy and variable resistance. Muscle power was normal, the tendon reflexes were symmetrical and normal, both plantar responses were flexor, and there was no defect of cutaneous or deep sensation. General examination was normal.

A CT scan in September 1979 revealed a high density lesion with an area of low attenuation within its anterior extent, which enhanced with contrast, in the external part of the right lenticular nucleus centred in the region of the putamen (fig 1). There was no displacement of the ventricular system. The following tests were reported as normal: EEG, skull and chest radiographs, copper metabolism, VDRL, liver and renal function, and blood count Plasma levels of glucose, urea, uric acid, cholesterol, calcium, phosphorus, and phospholipids also were normal.

The boy was treated sequentially with haloperidol ( 3 


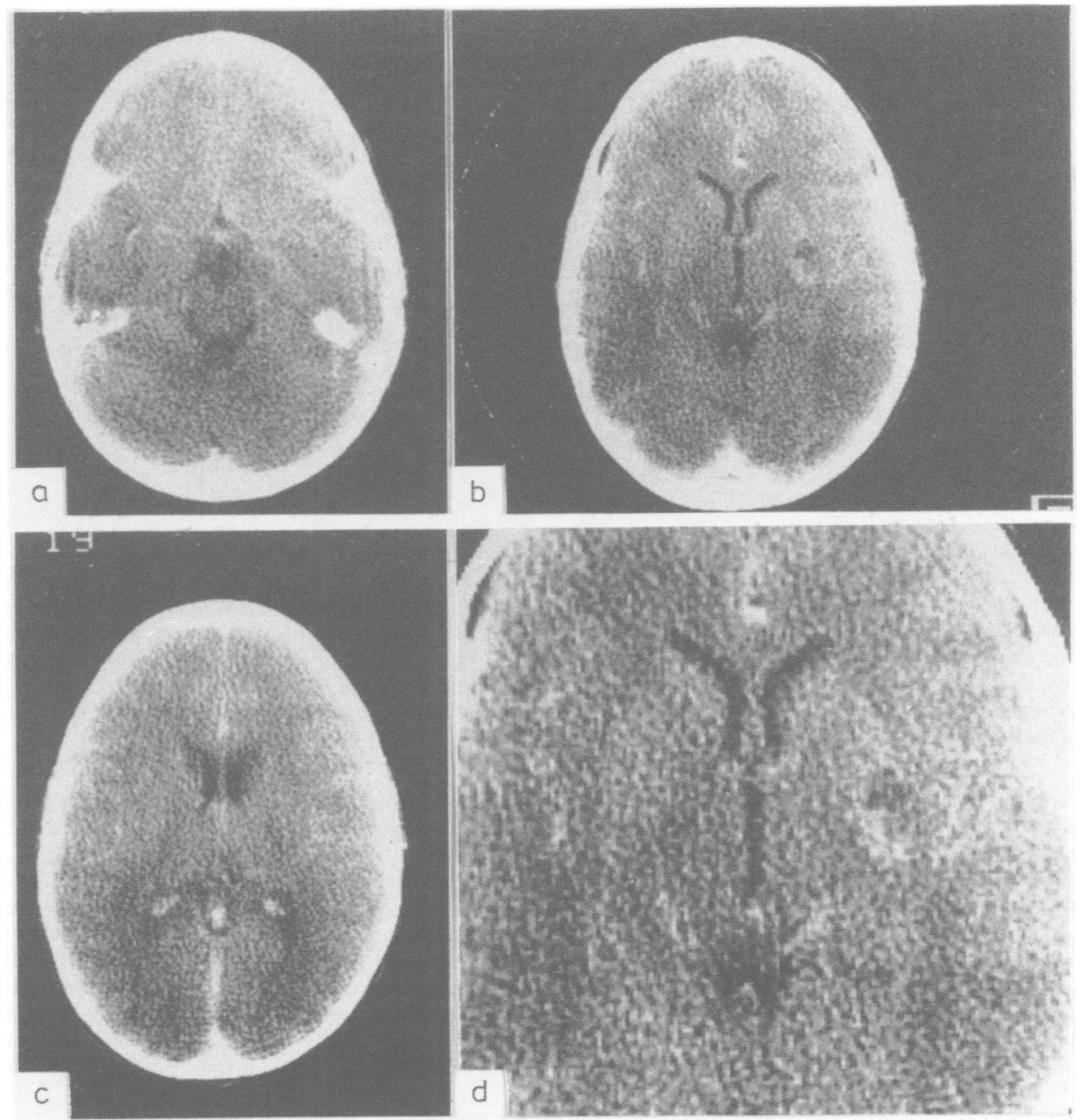

Fig 1 Horizontal CT scans after intravenous contrast injection. (a) Below the level of the third ventricle (no abnormality visible). (b) At level of third ventricle, showing a high density area, with a zone of low attenuation anteriorly in right putaminal area. (c) $12 \mathrm{~mm}$ above b (no clear abnormality visible). (d) Enlarged view of b. All scans were performed before radiotherapy or methotrexate treatment. The visible lesion corresponds in location to the area of maximum necrosis seen at necropsy (see fig 2). Note also that at the time of CT scans there was no hydrocephalus.

$\mathrm{mg} /$ day), benzhexol (25 mg/day), chlorpromazine $(75 \mathrm{mg} /$ day), and levodopa/carbidopa (750-75/day), without benefit. Six months after the onset of symptoms, he developed intense polyuria and polydipsia, drinking up to three litres of fluid each day. Diabetes insipidus was confirmed by measurement of plasma and urinary osmolality, and treatment with carbamazepine (300 $\mathrm{mg}$ daily) decreased the polyuria and polydipsia. This treatment also moderately improved the hemi-dystonia (30\% over control disability score). Two months later, however, the left arm became clumsier and walking was even more difficult. Examination at that time showed that he had signs in the left limbs of mild corticomotoneuron damage in addition to the hemi-dystonia. Examination of the cerebrospinal fluid now revealed neoplastic cells of uncertain type. At this point it was decided to begin a course of radiotherapy. 5000 rads directed to the right striatum were given in 25 sessions over five weeks. The left sided weakness subsequently resolved and there was some slight $(10 \%$ improvement in the hemi-dystonia). There was no deterioration over the next eight months, but then, 18 months after the onset of the illness, the hemi-dystonia worsened and the left hemi-paresis recurred. Intrathecal methotrexate was commenced, but four days later he died of mas- 

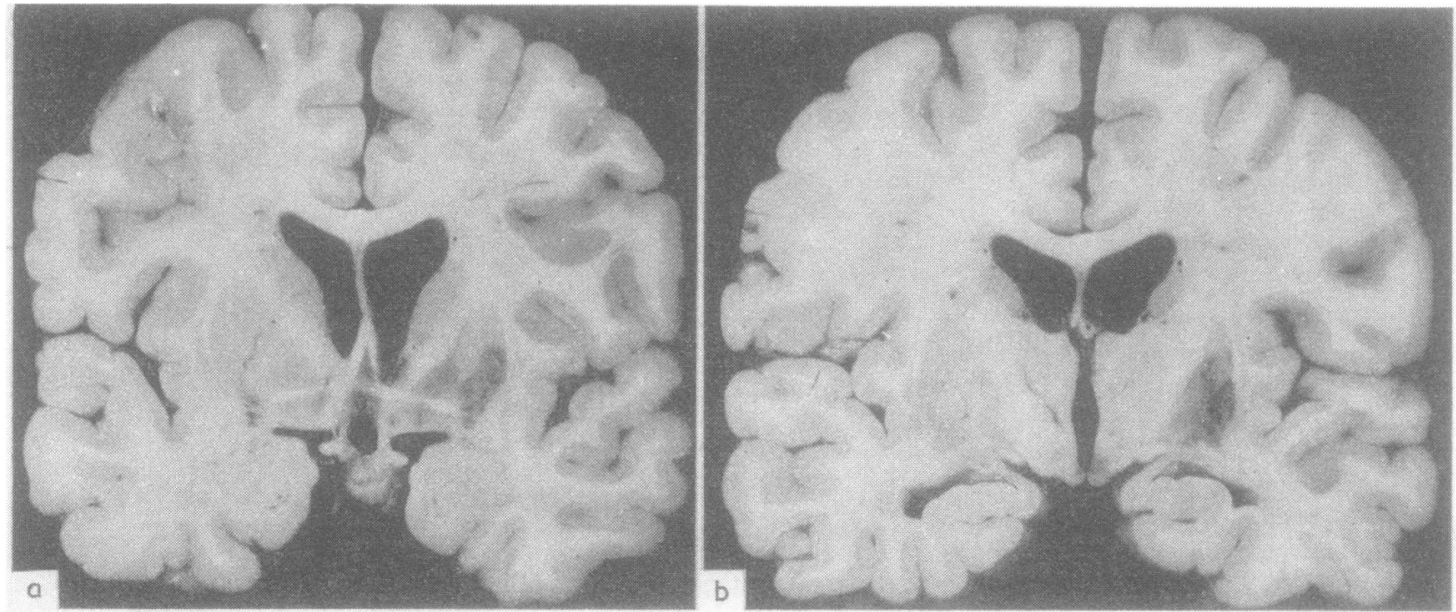

Fig 2 (a) Coronal section of the formalin foxed brain at the level of the anterior commissure. In the right putamen, in its inferior-lateral region, there is a cystic area of necrosis. There is infitration of tumour into both the external and internal segments of the globus pallidus. There also is an area of haemorrhagic necrosis subependymally at the inferior angle of the right lateral ventricle. There is hydrocephalus affecting not only the lateral ventricle, but also the anterior end of the third ventricle. (b) Coronal section at the level of the mammillary bodies. The area of cystic necrosis in the infero-lateral region of the putamen, and the surrounding tumour infitration again is shown. In addition, there is obvious macroscopic tumour in the right hypothalamus above the mammillary body. However, the subthalamus, thalamus, claustrum and caudate nucleus are normal bilaterally.

sive gastric haemorrhage.

Necropsy was done four hours after death. The brain weighed $1530 \mathrm{~g}$ and looked normal in external appearance. After formalin fixation, serial coronal slices (fig 2) revealed slight hydrocephalus and an area of necrosis in the inferolateral segment of the right putamen. There were obvious areas of tumour adjacent to the zone of necrosis in the putamen, and also in the right globus pallidus and right hypothalamus above the mammillary body. In addition, there was a zone of subependymal haemorrhage at the inferior angle of the right lateral ventricle. Histology showed the features of a low-grade astrocytoma, with only moderate hypercellularity and only a few undifferentiated cells (fig 3). The distribution of the tumour, as assessed in serial coronal sections, is shown in fig 4 . The area of cystic necrosis was confined to the infero-lateral putamen. The solid tumour was confined histologically within the basal ganglia to the putamen, but extended perivascularly into the globus pallidus (pars externa and interna). There also was slight perivascular extension medially into the internal capsule and dorsally into the subependymal zone of the roof of the right lateral ventricle. Solid tumour was present

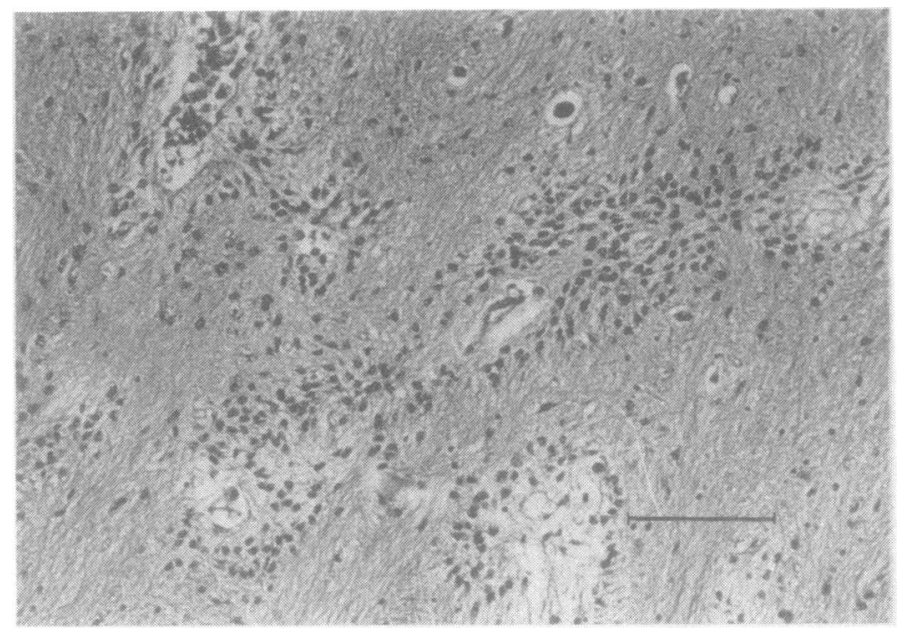

Fig 3 Histology of the tumour. An area of globus pallidus is shown to illustrate both the cellular nature of the tumour (astrocytoma), and its perivascular distribution in otherwise normal brain in this area. The scale marker is $100 \mu$. Haematoxylin-eosin. 

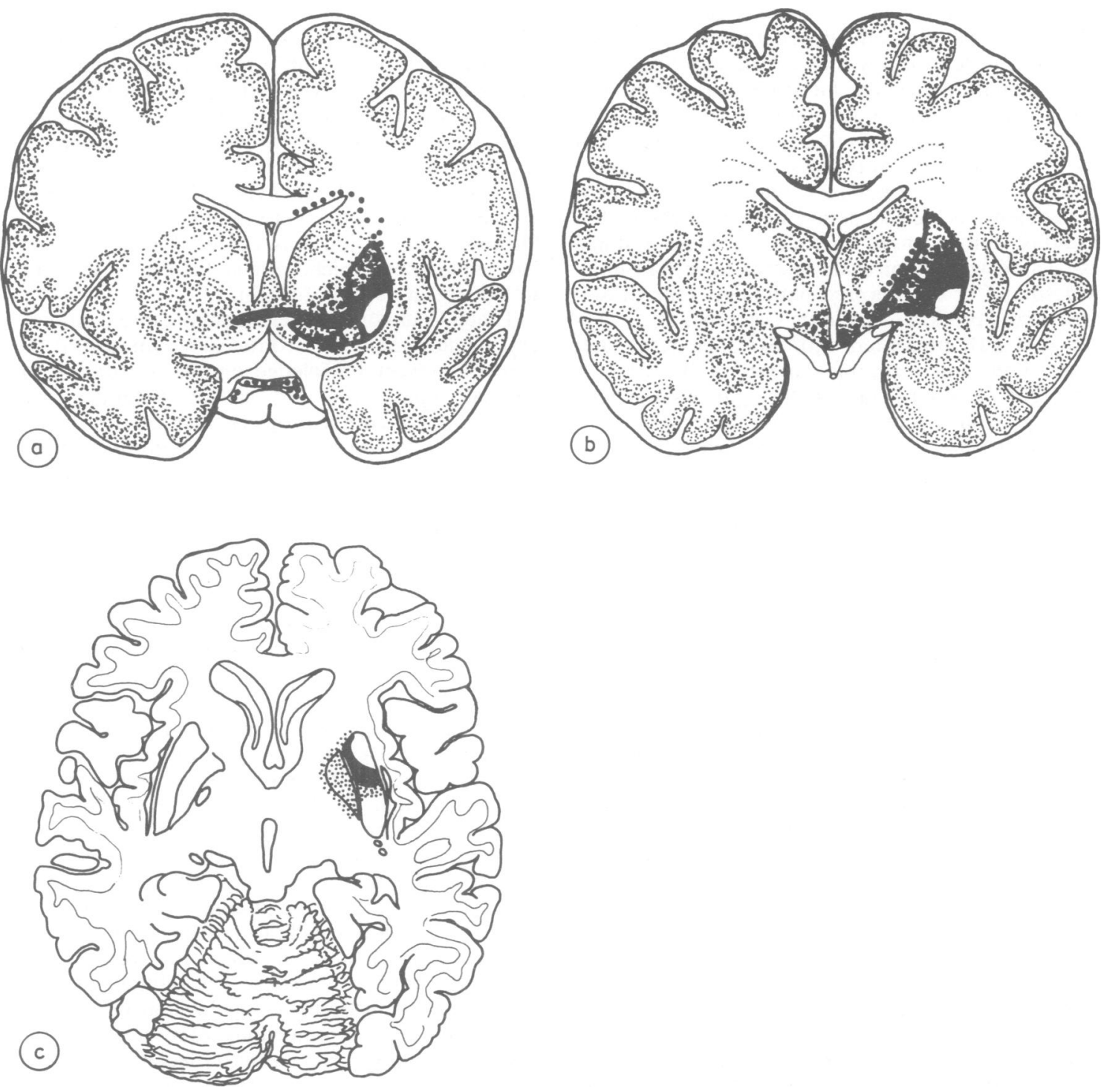

Fig 4 Histological distribution of the tumour shown in diagrams of sections of the brain. (a) Coronal at level of anterior commissure, (b) Coronal at level of infundibulum, and (c) Horizontal at level of third ventricle. The solid white zone indicates the area of cystic necrosis in the right putamen. The posterior half of the putamen (also in white in the horizontal diagram) was normal histologically. The solid black zone indicates the areas of brain replaced by solid tumour. The dotted black zones indicate areas of brain containing perivascular tumour.

also in the hypothalamus, bilaterally, in the anterior commissure and in the optic chiasma. The thalamus, claustrum, subthalamus, substantia nigra (zona compacta and reticulata), superior colliculus, red nucleus and brain stem were normal, as were the motor cortex and cerebellum, both macroscopically and microscopically.

\section{Discussion}

Pathologically well-documented cases of focal brain lesions causing focal or hemi-dystonia have been described but rarely. Urechia et $a^{3}$ published the case of a 14-year-old boy with a tumour in the 
putamen, caudate nucleus and globus pallidus. Chromatolysis and fatty degeneration of the subthalamic nucleus also was observed. Another localised lesion producing focal dystonia was reported by Lopez Aydillo and Sanz Ibañez, ${ }^{4}$ who studied a 56-year-old female diabetic patient with dystonia of the left arm of vascular origin secondary to complete necrosis of the right putamen and head of the caudate nucleus, as well as demyelination of the globus pallidus. Other cases of focal or hemi-dystonia associated with local brain lesions have had more widespread or complicated pathology. One of Sciarra and Sprofkin's ${ }^{5}$ patients (case No 7), a 15year-old girl, presented with dystonia of the left upper limb and jerky movements of the toes. A large glioblastoma invading the right basal ganglia from the frontal lobe was found at necropsy. Gordin $^{6}$ described a 23-year-old man who developed torticollis with the chin deviated to the right, twisting of the trunk to the right with excessive lordosis and hemi-dystonia of the left limbs. At necropsy, extensive changes were found in the right caudate nucleus, putamen and, to a lesser extent, the external segment of the globus pallidus. These consisted of neuronal degeneration and glial proliferation, with perivascular gliosis, and were attributed to "a slight encephalitic affection". This diagnosis raises a question of more diffuse pathology, although none was described. In all of the remaining published cases of symptomatic focal dystonia studied pathologically that we have had the opportunity to review in detail, the dystonia had emerged as a late sequela of acute vascular hemiplegia, ${ }^{7-10}$ or was accompanied by additional neurological signs from its onset, ${ }^{510-12}$ indicating initial extensive brain damage.

A number of cases of focal dystonia of one limb, or of hemidystonia, have been described since the advent of the CT scan, with lesions in the region of the contralateral basal ganglia, either vascular ${ }^{13-15}$ or traumatic ${ }^{16}$ in origin. However, the exact localisation and extent of such lesions is difficult to establish by radiological techniques alone. Bilateral putaminal lesions also may be associated with dystonia. For instance, such pathology is found in Wilson's disease and also occurs in Leigh's disease, in both of which occasional patients may present with typical dystonia. However, the pathology in such cases undoubtedly is more widespread, so that exact clinico-pathological correlation is difficult.

The localisation of the tumour in our patient allows accurate clinico-pathological correlation. Typical dystonia on action and at rest, with mobile spasms showing co-contraction of antagonist muscles and overflow of muscle activity, was present in the left limbs for several months as the only neurological sign. Five months after the onset of the illness, a CT scan revealed a focal lesion in the external part of the lentiform nucleus, corresponding to the putamen. Even at the end of the illness, 18 months after the dystonia first began, the maximum tumour necrosis was in the infero-lateral putamen. By then, however, there had been tumour spread into the globus pallidus, hypothalamus (with diabetes insipidus), optic chiasm, and internal capsule. However, the lesion was centered in the inferolateral putamen, which, from all the evidence, seems to have been its site of origin.

That a lesion of the putamen may cause contralateral hemidystonia is of considerable interest in the light of present knowledge on the distribution of cortical inputs into the caudate-putamen. The sensori-motor cortex projects, in primates, to the putamen, with some degree of somatotopic organisation. ${ }^{17-19}$ Kunzle, ${ }^{17}$ for example, has identified projections from motor cortex to putamen in the monkey, with the leg area anterodorsally, the face area postero-inferiorly, and the arm area intermediate between the two. There is some resemblance between this organisation and the site of dystonia in our patient and the site of the lesion at necropsy.

In the light of this case, and the few published reports reviewed above, focal limb dystonia may be regarded as secondary to a lesion of the lenticular nucleus and probably the putamen in particular. This is not to suggest that some other brain structures may not also be implicated in other cases of dystonia in man. In all these cases of symptomatic dystonia, dystonia may be regarded as a release phenomenon due to disinhibition of some other nucleus or area within the basal ganglia or elsewhere. The major outputs of the putamen are to both segments of the globus pallidus (which were damaged in our patient) and to substantia nigra (zona reticulata) which was spared. The subthalamic nucleus, thalamus, superior colliculus and brain stem, all of which receive projections from the globus pallidus or substantia nigra zona reticulata, also were intact. Which of these areas are involved by disinhibition as a result of the putamen lesion to cause the dystonia cannot be decided. Whether or not a similar mechanism operates at the biochemical level to account for the motor abnormalities present in patients with idiopathic or primary torsion dystonia is not known. However, cases such as those reviewed here suggest that biochemical examination of brains from patients with idiopathic dystonia should include careful dissection of discrete areas of the putamen and globus pallidus, and of their sites of projection.

The authors are grateful to Dr JL Zubieta 
(Neuroradiology Unit, Clinica Universitaria) for his expert comments and Mrs M Obeso for skilful assistance.

\section{References}

' Zeman W. Pathology of the torsion dystonias (dystonia musculorum deformans). Neurology, 20 (part 2) 1970:79-88.

${ }^{2}$ Zeman W, Whitlock CC. Symptomatic dystonias. In Vinken PJ, Bruyn GW eds.), Handbook of Neurology Amsterdam: Elsevier, 1968, Vol. 6. 545-63.

${ }^{3}$ Urechia CI, Dragomir L, Usinievici G. Spasme de torsion unilatéral causé par une tumeur cérébrale. Conf Neurologica 1942-3;5:271-80.

${ }^{4}$ López Aydillo NR, Sanz Ibañez J. A propósito de un caso de distonía de torsión (variante miostática o paralítica) en una diabética glucosúrica. Trabajos Inst Cajal Inv Biol 1956;48:81-108.

${ }^{5}$ Sciarra F, Sprofkin B. Symptoms and signs referable to the basal ganglia in brain tumour. Arch Neurol Psychiat 1953;69:450-61.

- Gordin R. A case of unilateral torsion dystonia: A clinico-histological study. J Nerv Ment Dis 1939;90:344-57.

7 Denny-Brown D. The Basal Ganglia. London: Oxford University Press, 1962:55-6.

${ }^{8}$ Oppenheimer DR. A case of striatal hemiplegia. $J$ Neurol Neurosurg Psychiatry 1967;30:134-9.

9 Dooling EC, Adams RD. The pathological anatomy of post-hemiplegic athetosis. Brain 1975;98:29-48.
${ }^{10}$ Garcin R. Syndrome cerebello-thalamique par lesion localiseé du thalamus. Rev Neurol (Paris) 1955;93:143-9.

"Austregesilio A, Borges-Forte A. Sur un cas d'hemichorée avec lesion du moyau caudé. Rev Neurol (Paris) 1937;67:477-88.

${ }^{12}$ Chorobski J. Involuntary movements in patients with intracranial tumours. Arch Neurol 1962;6:27-56.

${ }^{13}$ Demierre B, Rondot P. Dystonia caused by putaminocapsulo-caudate vascular lesions. J Neurol Neurosurg Psychiatry 1983;46:404-9.

14 Russo LS. Focal dystonia and lacunar infarction of the basal ganglia. Arch Neurol 1983;40:61-2.

15 Traub M, Ridley A. Focal dystonia in association with cerebral infarction. J Neurol Neurosurg Psychiatry 1982;45:1073-4.

${ }^{16}$ Brett EM, Hoare RD, Sheehy MP, Marsden CD. Progressive hemi-dystonia due to focal basal ganglia lesion after mild head trauma. $J$ Neurol Neurosurg Psychiatry 1981;44:460.

${ }^{17}$ Kunzle H. Bilateral projections from precentral motor cortex to the putamen and other parts of the basal ganglia. An autoradiographic study in Macaca Fasicularis. Brain Res 1975;88:195-209.

${ }^{18}$ Künzle H. Projections from the primary somatosensory cortex to basal ganglia and thalamus in the monkey. Exp Brain Res 1977;30:481-92.

19 Jones EG, Coulter JD, Buxton $\mathrm{H}$ et al. Cells of origin and terminal distribution of cortico-striatal fibres arising in the sensory-motor cortex of monkeys. J Comp Neurol 1977;173:53-80. 